\title{
A proactive approach to the conservation of historic and cultural Heritage: the HeritageCare methodology
}

\author{
Maria José Morais, Maria-Giovanna Masciotta, Luís F. Ramos, Daniel V. Oliveira, Miguel Azenha, \\ Eduardo B. Pereira, Paulo B. Lourenço
}

ISISE, Institute of Science and Innovation for Bio-Sustainability (IB-S), Department of Civil Engineering, University of Minho, Guimarães, Portugal

\section{Teresa C. Ferreira}

Center for Studies in Architecture and Urbanism, School of Architecture, University of Porto, Portugal

\author{
Paula Monteiro
}

Centre for Computer Graphics, Guimarães, Portugal

Contacting author: mariajcmorais@gmail.com

\begin{abstract}
Presently, no systematic policy for the preventive conservation of the built cultural heritage exists in South-West Europe. Current approaches for inspection, diagnosis, monitoring and reactive conservation are normally intermittent, unplanned, expensive and lack methodical strategy. The available financial resources are scarce and are mostly addressed to listed buildings. Besides, owners and stakeholders often reveal reluctance to invest in preventive conservation and maintenance programs. In view of these considerations, and driven by the principle "prevention is better than cure", the HeritageCare project has developed a system for the preventive conservation and maintenance of the built heritage. The main aim of this paper is to briefly describe the methodology, including its three levels of service, and present the main results of the implementation and validation of the service level 1 on a case study belonging to the Portuguese architectural heritage.
\end{abstract}

Keywords: preventive conservation; cultural heritage; inspection.

\section{Introduction}

Currently in South-West Europe, there is no systematic policy for the preventive conservation of the built cultural heritage, being the conservation strategies mainly focused on reactive conservation and restorative treatments, often carried out when damage is severe [1,2]. Also, there is an absence of appropriate preventive conservation and maintenance and plans aiming at medium/long term. This leads to considerable investments of societal and financial resources. Finally, there is a lack of information about the real benefits associated with regular inspections and preventive measures $[3,4]$.

Moreover, the existing damage atlases are oriented according to specific regions, types of material or causes. The terms used, as well as the 
ways damage is identified, are often incomplete and non-uniform, leading to possible misunderstandings [5-7].

Influenced by the Monumentenwacht concept $[1,8-11]$ and aiming at improving this concept, the HeritageCARE project (monitoring and preventive conservation of historic and cultural heritage in Southwest Europe) has been launched in 2016 [1214]. It is a three-year project composed of eight partners distributed among three partner countries, Portugal, Spain and France, coordinated by the University of Minho and funded by the European Regional Development Fund (ERDF) within the Interreg-SUDOE program.

The following sections describe briefly the methodology developed within the project, as well as its implementation in a case study, the Ducal of Palace in Guimarães (Portugal).

\section{Methodology}

The HeritageCare methodology [12] relies on a preventive management three-level system, based on systematic inspections and monitoring of built heritage supported by the latest advances in digitization and smart technologies (such as laser scanning, photogrammetry, Historic Building Information Modelling and Augmented Reality). Service Level 1 provides owners (or managers) with a feasible, low-cost and rapid condition screening of the conservation status of the building. Service Level 2 aims at integrating the information collected in Service Level 1 with an in-depth condition assessment of the conservation status of the main assets present in the building, as well as relevant monitoring data records. The owner can visualize all this information navigating across his/her building through a virtual tour, having also access to monitoring control parameters in realtime. In Service Level 3 the owner (or manager) can have the building managed through a threedimensional BIM model, where all meaningful information and input data collected from the previous service levels are also integrated.

Within Service Level 1, also called StandardCare, a digitized inspection of the building is carried out by two professionals (trained architects or engineers). This condition survey encompasses all the building, from top to down, site, outside and inside, including the installations and a rapid condition screening of the integrated and movable assets. Unlike traditional inspection routines, the HeritageCare inspection is carried out making use of fillable e-forms through a mobile application, being all the collected data stored in a structured database [13].

In a preliminary phase, a survey was conducted looking at: (1) existing building typologies and construction systems; (2) typical damages and deterioration processes found in the South-West Europe built heritage and assets with cultural and historical value; (3) damage classifications; (4) condition grading scales; (5) inspection forms; (6) initiatives/systems working in the field of preventive conservation; (7) aspects to consider during the process of inspection and preventive conservation management.

The existing information was incomplete, not uniform or not adaptable to the reality of the different countries inside the South-West European region. Therefore, the project started with the creation of basic supporting information, such as: (1) classification of building typologies and assets with cultural and historical value; (2) damage atlas for buildings and assets, where a unique and uniform damage classification was created for both buildings and assets, along with a systematic structure of parameters considered necessary to properly characterize each damage; (3) condition and risk classification grading scales; (4) structure of the inspection forms and management process for Service Level 1. For more detailed information the reader is referred to [15].

The web platform, the supporting database and the mobile application were developed by Centre for Computer Graphics (CCG), partner of the project, according to the specifications decided within the consortium.

The methodology and the tools were implemented and validated through case studies in each country of the consortium. The case studies were selected taking into account the great variety of built heritage typologies present in the SUDOE territory, as well as their different conservation stages, with the aim of evaluating the applicability of the methodology to different scenarios. 


\section{Case study of Ducal Palace}

\subsection{Description}

The case study here presented is the Ducal Palace in Guimarães, Portugal (see Figure 1), a three-story masonry building dated from the $15^{\text {th }}$ century and classified as National Monument. Nowadays with an important restoration in the $X X$ century, this building is hosting a museum, serves as presidential residence and receives several cultural initiatives.

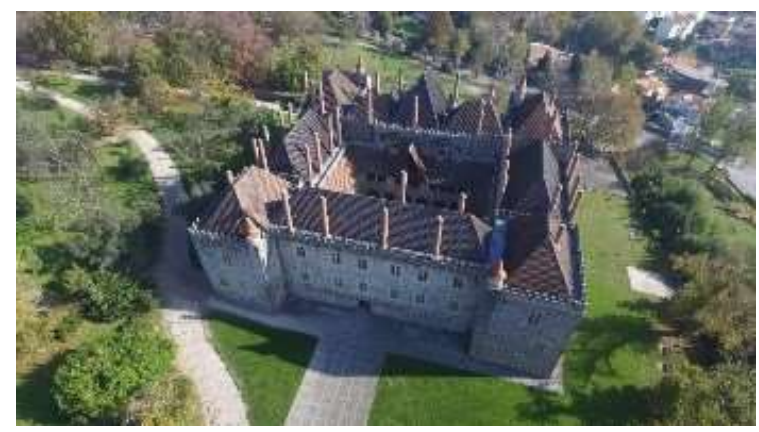

Figure 1. Ducal Palace in Guimarães (Portugal).

The Service Level 1 follows a protocol divided into three steps, namely pre-, on-site and postinspection, which are presented in the next sections.

\subsection{Pre-inspection}

The pre-inspection step consists in the collection of the existing information concerning the building. It starts with a first contact with the owner of the building, or the manager, through a questionnaire to get a better insight of his/her property status regarding the use, state of conservation and major maintenance problems experienced. There is the option to perform this task using an online platform.

The collected data is uploaded to the HeritageCare database through a web platform in two groups of information: (i) building identification, where the data concerning general information, ownership, construction and geometry is stored, and where the plans and elevations are divided into rooms to be used during the on-site inspection; (ii) the second group includes the management information, where the data concerning construction and integrated and movable assets is included.
The last step consists in the attribution of a weight to each group of building items, according to its importance for the building and according to a division established by the partnership. The building is divided in four item groups that, in turn, are divided into different items, which are divided into different sub items (construction elements). For more detailed information, the reader is referred to [12].

Figure 2 presents an excerpt of the web-platform for the pre-inspection phase, where it is possible to upload data associated to the building. For the building identification, the following information is included: (i) general object (identification, function, etc.); (ii) information about the owner/manager/tenant; (iii) construction (description of the object, main materials, main dimensions, site conditions and period of construction); (iv) map information (urban plan, maps and elevations with the division in rooms and facades already prepare for the inspection).

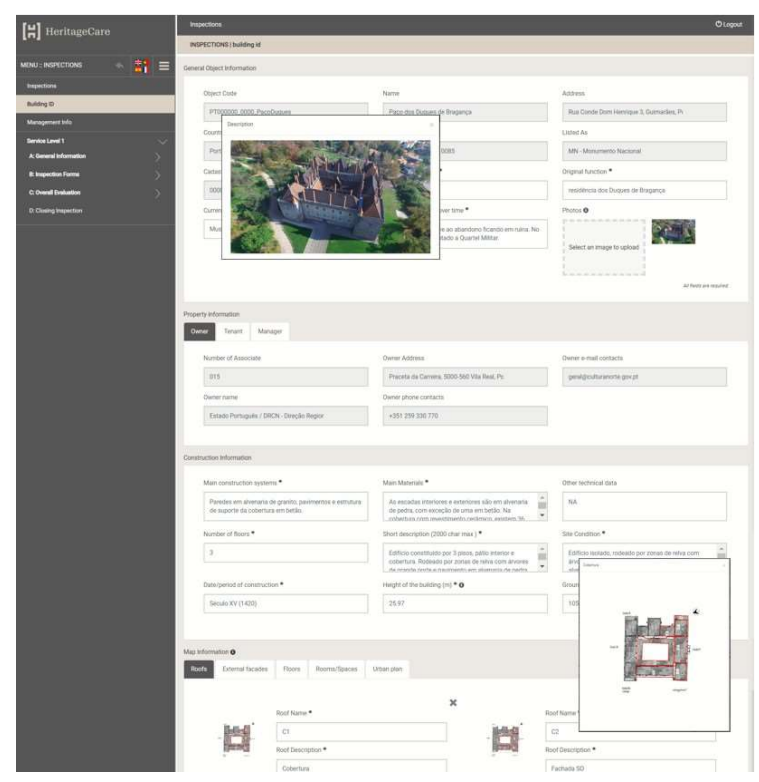

Figure 2. Web platform - Pre-inspection phase used for collecting the data concerning the Ducal Palace.

As for the management information, it encompasses: (i) construction (historical information and construction phases, previous inspections/interventions/maintenance actions); (ii) integrated and movable objects (quantitative information and other aspects). Figure 3 presents an example of the attribution of the condition weighting factors. 


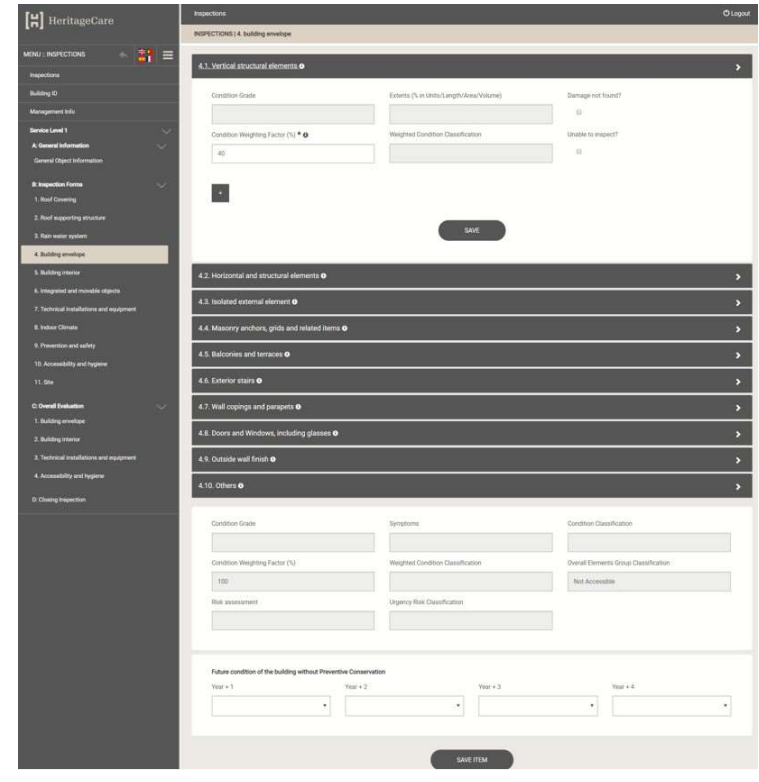

Figure 3. Web platform - example of preinspection phase for the attribution of the condition weighting factors.

\subsection{On-site-inspection}

The on-site inspection is carried out by filling in an inspection e-form, accessible through a mobile application by using a tablet, and the plans and elevations to represent the damages observed according to the damage classification and the damage code developed. During the on-siteinspection, inspectors carry with them personal safety equipment (e.g. helmets, jackets) and some tools (e.g. digital camera, binoculars, laser distance meter, artificial light, crack meters, chisel, ladder and hammer) in case they need to verify some parameters or to make small cleaning works, easy repair works or even urgent measures that cannot be postponed.

The mobile application follows the same approach as the web-platform but adopting a structure that is more user-friendly for on-site use, being possible to have access to all the data uploaded during the previous stage that is organized in three parts. The first part is dedicated to general information, including the access to the data concerning the building that originated from the pre-inspection step, as well as to adding the conditions found during the on-site inspection. In the second part, for each building item, damage is documented based on the damage atlas developed, accessed through the mobile application. Also, according to the grading scale system, a qualitative/quantitative index is attributed to each of the inspected items, and according to the role played by each element within the building this index is weighted according to the extent of damage. Finally, consequences of the damages identified, and recommendations are provided. The mobile application allows to take photos of the identified damages. The access to the damage atlas during the on-site-inspection allows inspectors to perform the correct identification of damages, without the need for a post-processing phase in office. The mobile application also allows inspectors to generate audio records. The third part includes the synchronization of the mobile application with the web-platform, so that the data collected during the on-site inspection can be transferred to the database. The use of a fillable inspection e-form synchronized with the webplatform allows this step to be faster and accessible, without the risk of missing any important part of the inspections.

For the Ducal Palace, problems related to humidity were observed inside the building, as a result of infiltrations at the roof covering and at the windows and doors. Also significant temperature oscillations have been reported by Ducal Palace staff members working indoors. Degradation of the stone due to the presence of cement mortars was also observed (both inside and outside the building), more evident on the first floor, SE façade. Also, at this façade and at the SW façade, cracks were visible both inside and outside of the building.

Figure 4 shows the part of the process related to the identification of the damages for one of the sub building items: the vertical structural elements from the building envelope.

During the inspection, the interview with the manager team, as well as with the people working at the Palace was essential to complement the data collected. The information typically collected by means of interviews with the owners or frequent users of the indoor spaces are crucial to access information that is hardly obtained by any other means, especially concerning information related to the historical data prior to the inspection of the buildings. 

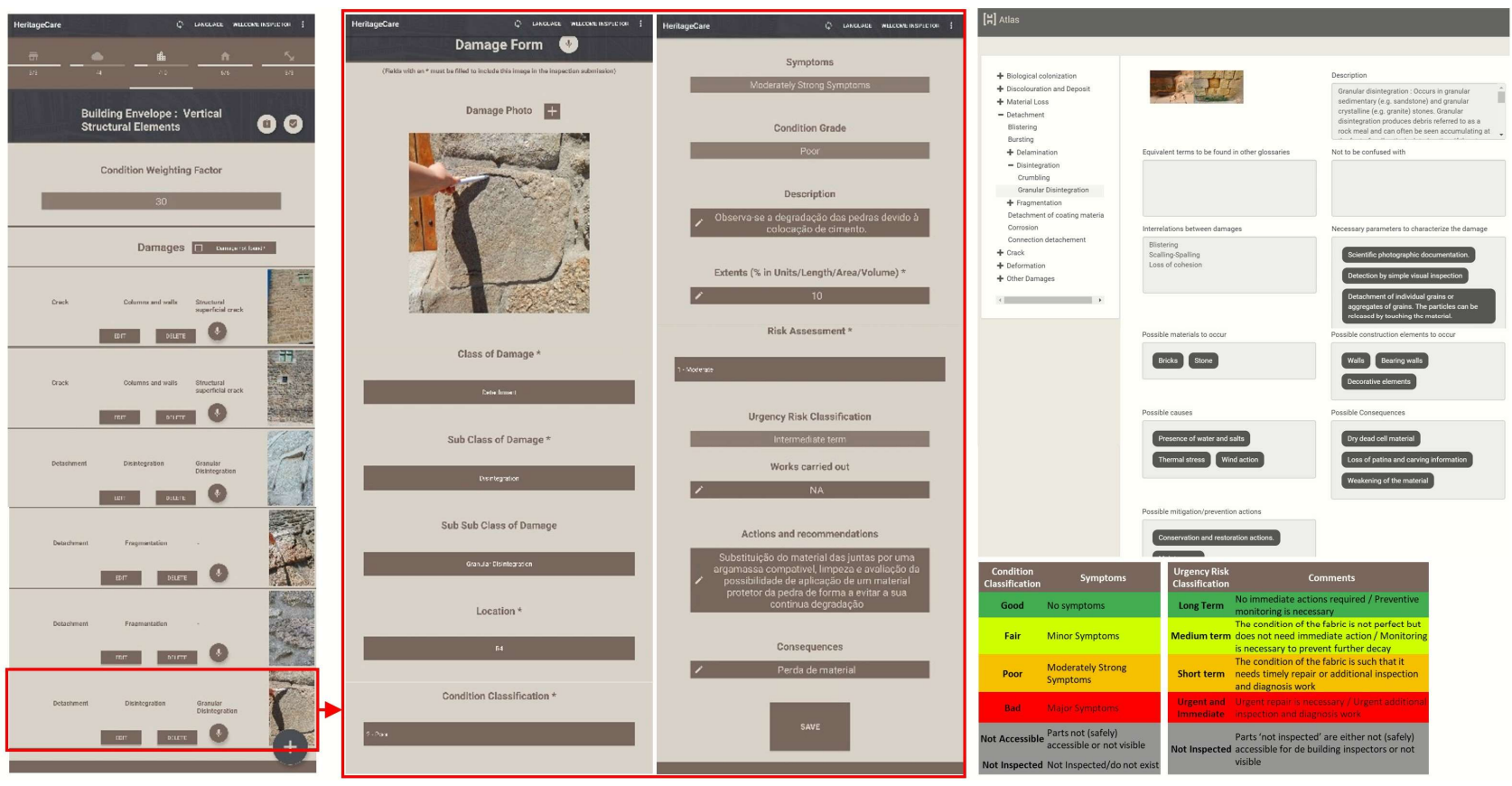

Figure 4. On-site-inspection phase with the mobile application (from left to right: list of some of the damages for the vertical structures elements; parameters used to identify one of the damages observed; damage atlas and grading scale system).

\subsection{Post-inspection}

The post-inspection is a back-to-office process, where the information uploaded during the previous stage is analysed and, if necessary, updated. The plans and elevations with the damages are updated and the final remarks are registered.

For each group of building elements and according to the condition grade, risk assessment and damage extension, an overall evaluation is established, which supports the subsequent decision about the most important recommendations and necessary conservation and maintenance actions to undertake in the short, medium and long term to prevent further decay. The condition grade of each building item corresponds to the condition grade of the most damaged part (see Figure 5).

Also, the future condition of the building is established for the next four years if no measures are undertaken.
The output of this stage is the automatic condition report for the owner (or manager), through which it is possible to have access to: (1) the condition assessment and recommendations for short, medium or long term maintenance, track record of preventive conservation actions and schedule of future maintenance actions; (2) a description of each of the damages through their condition, location, consequences, priority of intervention and works carried out; (3) links to the guidelines for good practices on preventive conservation developed by the partnership.

\subsection{Building management}

The condition report is delivered to the owner (or manager) of the building, followed by a questionnaire with the aim of collecting his feedback concerning the inspection. The owner's willingness to implement the recommendations provided in the condition report is also recorded. 

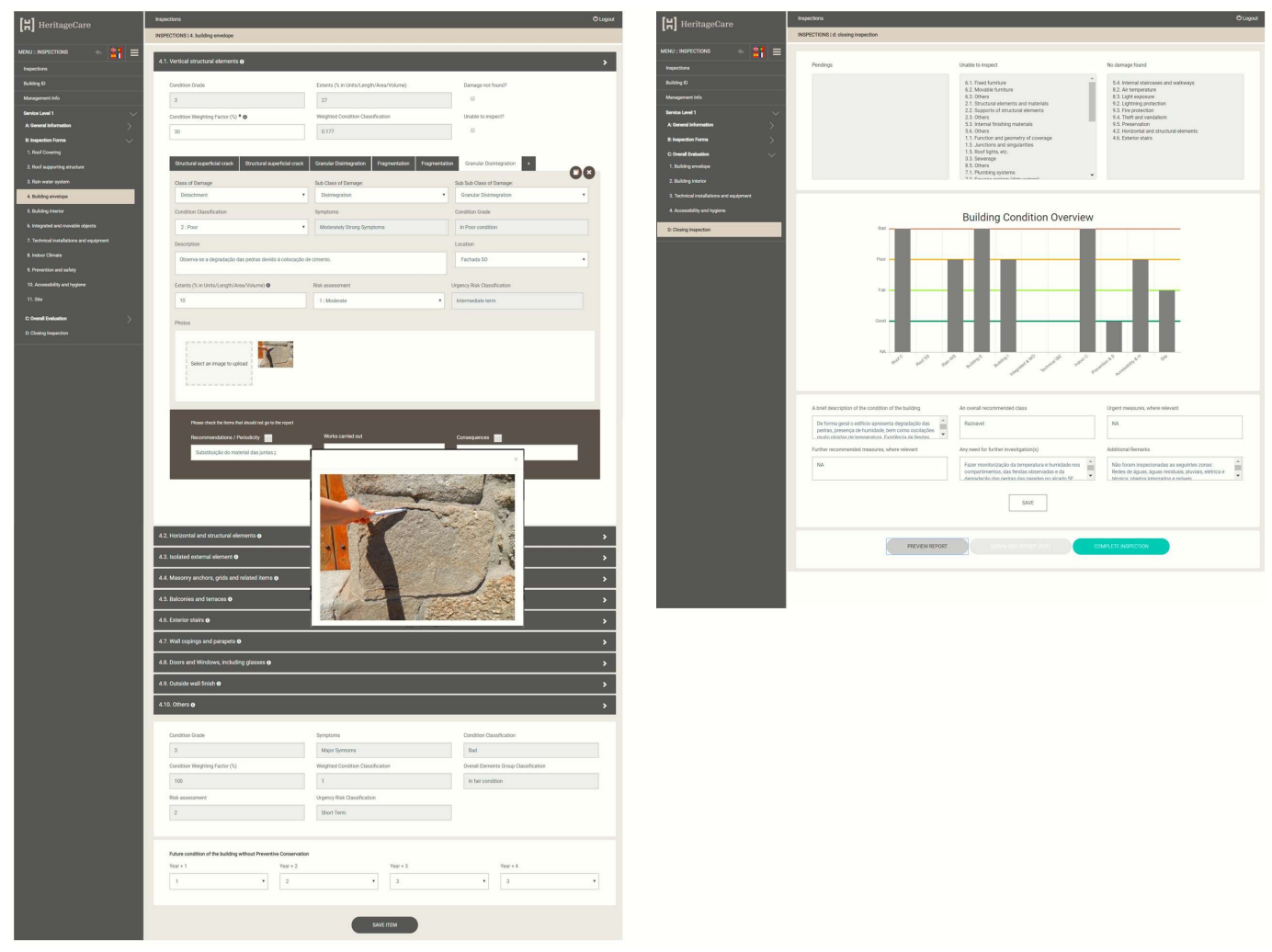

Figure 5. Post-inspection phase using the web platform (from left to right: building item with the identification of the damages for each sub building item and for the building item; condition overview of the building).

The owner (or manager) can have access to all the data concerning the building through a restricted access to the database, accessible through the project website (see Figure 6). Also, in case of a serious or urgent problem, the owner (manager), as well as national Heritage Authorities receive a warning message, being advised to contact an expert in order to perform an in-depth inspection and to plan a prompt intervention. Information about construction companies operating according to the best practices and with recognized experience is also provided.

According to the type of building and its conservation status, the inspection is done periodically. This will allow to trace the conservation status of the building over time. The re-inspection follows the same procedures of the previous one, so it is possible to successively compare building conditions, highlighting whether or not the recommendations provided were followed. During the phase of on-site-inspection, the inspectors can have access to information from the previous inspection through the mobile application.

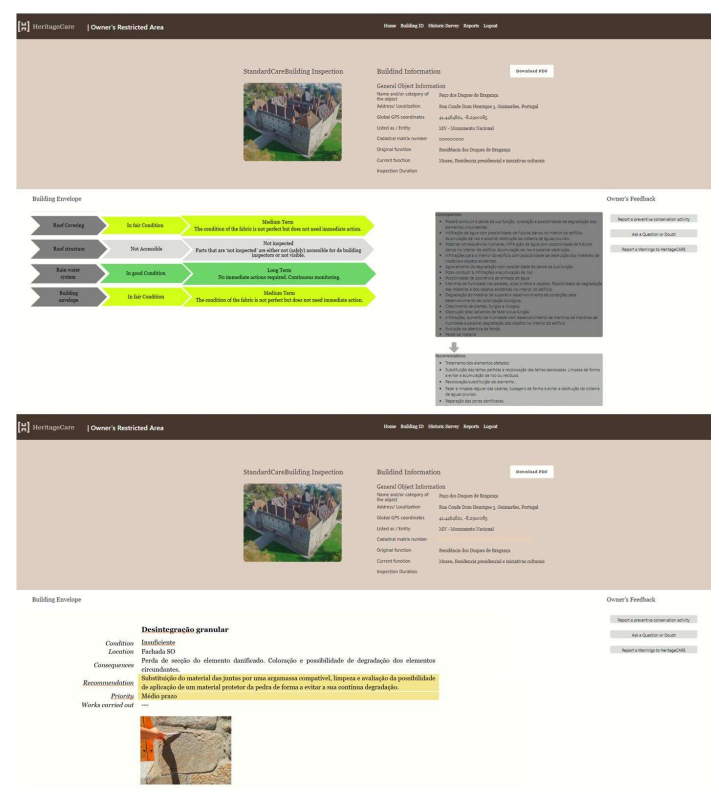

Figure 6. Owner (or manager) area for the Ducal Palace, where is possible to access the building information. 


\section{Conclusions}

The final goal of the HeritageCare project is the creation of a non-profit self-sustaining entity that will implement the HeritageCare methodology with tailored tools providing for an affordable way for owners (or managers).

The implementation and validation of the first service level allowed to improve the methodology and tools developed, as well as to improve the methodology proposed for the next two service levels.

After receiving the condition report, the manager of the present case study agreed with the recommendations proposed. As a consequence, some of these have already been implemented, such as the placement of a monitoring system to measure temperature, luminosity, humidity and the presence of xylophagous, aiming at understanding the causes and the consequences of some of the damages observed during the on-siteinspection. Additionally, crack meters will be placed to monitor the cracks observed inside and outside the building. The re-inspection phase is scheduled to take place in two years, and this inspection will allow to understand if all the recommendations were followed as well as to observe the effect of those recommendations, and reassess the condition of the building. Following this proactive approach, it will be possible to prove that the existence of a structured preventive conservation process results in benefits to owners in terms of the health condition of his property and also in financial terms. Furthermore, it will be possible to understand if there are additional aspects in the process that need further improvement.

\section{Acknowledgments}

This work was carried out within the scope of the project HeritageCare (SOE1/P5/P0258), co-funded by the Interreg-Sudoe/FEDER program and included in the research activities of the ISISE Research Centre, also financed by FEDER funds through the Competitiveness Factors Operational Program - COMPETE and by national funds through FCT - Foundation for Science and Technology within the scope of the project POCI-01-0145FEDER-007633.

Authors would like to express their gratitude to the Centre for Computer Graphics, HeritageCare partner, for the development of the platform (and supporting database) and the mobile application, outputs of the project HeritageCare.

Authors would like to express their gratitude to the Santa Maria de la Real Foundation, HeritageCare partner, for the placement of the monitoring system at the Ducal Palace, Guimarães, Portugal.

\section{References}

[1] K. Van Balen, Challenges that preventive conservation poses to the cultural heritage documentation field, in: Int. Arch. Photogramm. Remote Sens. Spat. Inf. Sci. ISPRS Arch., 2017: pp. 713-717. doi:10.5194/isprs-archives-XLII-2-W5-7132017.

[2] K. Van Balen, A. Vandessand, Innovative built heritage models based on preventive and systematic approaches, in: A. Balen, K. Van; Vandessand (Ed.), Int. Conf. Innov. Built Herit. Model. Prev. Syst. (CHANGES 2017), Leuven, 2017: p. 8. https://www.taylorfrancis.com/books/e/9 781351014786.

[3] A. M. Forster, B. Kayan, Maintenance for historic buildings: a current perspective, J. Qual. Maint. Eng. 27 (2009) 273-280. doi:10.1108/02630800910971347.

[4] H. Wirilander, A Key Method to Ensure Cultural Heritage Authenticity and Integrity in the Preservation Process By Heidi Wirilander, (2012). http://www.econservationline.com/.

[5] Structural damage in masonry: Developing diagnostic decision support, n.d. https://pdfs.semanticscholar.org/59aa/082 b7ee20fe17a3931c9d4c60a9960a4a051.pd f?_ga=2.194603182.222181291.154879096 3-648407238.1548790963 (accessed September 29, 2018).

[6] L. Binda, L. Franke, Mateus (K.U.Leuven, Raymond Lemaire Centre for Conservation, 
Leuven (Belgium))., (n.d.) 1687-1693.

[7] V. Vergès-Belmin (Ed.), ICOMOS-ISCS: Illustrated glossary on stone deterioration patterns, $\quad$ (2008) 78p. doi:10.3109/14767058.2010.497569.

[8] V. Meul, A. Stulens, The integral approach of Monumentenwacht Vlaanderen: a model for implementing participatory preventive conservation for historic interiors, 2010.

[9] S. N. H. Heinemann, The role of Monumentenwacht 40 years theory and practice in the Netherlands, in: A. Balen, Koen Van; Vandesande (Ed.), Innov. Built Herit. Model. Ed. Contrib. to Int. Conf. Innov. Built Herit. Model. Prev. Syst. (CHANGES 2017), Leuven, 2017. https://www.crcpress.com/Innovative-

Built-Heritage-Models-Editedcontributions-to-the-International/BalenVandesande/p/book/9781138498617.

[10] N. Van Roy, E. Verstrynge, A. Vandesande, K. Van Balen, Implementation of maintenance systems: Identification of maintenance practices within the Monumentenwacht model in Belgium, in: A. Balen, Koen Van; Vandesande (Ed.), Innov. Built Herit. Model. Ed. Contrib. to Int. Conf. Innov. Built Herit. Model. Prev. Syst. (CHANGES 2017), Leuven, 2017. https://www.crcpress.com/InnovativeBuilt-Heritage-Models-Editedcontributions-to-the-International/BalenVandesande/p/book/9781138498617.

[11] N. Cebron, L. Koenraad, Preventive conservation and maintenance of architectural heritage as means of preservation of the spirit of place, in: 2001: pp. 1-8.

[12] HeritageCARE, General methodology for the preventive conservation of cultural heritage buildings, published document, HeritageCARE (Report of the Project Activity 1.2), 2017.

[13] HeritageCARE, Technical requirements for the tools to develop on the HeritageCare project, Unpublished document,
HeritageCARE (Report of the Project Activity 1.3), 2017. http://heritagecare.eu/.

[14] L.F. Ramos, M.G. Masciotta, M.J. Morais, M. Azenha, T. Ferreira, E.B. Pereira, P.B. Lourenço, HeritageCARE : preventive conservation of built cultural heritage in the South-West Europe, in: A.V. Koenraad van Balen (Ed.), Innov. Built Herit. Model. Ed. Contrib. to Int. Conf. Innov. Built Herit. Model. Prev. Syst., 1st ed., Leuven, Belgium, 2018.

https://www.crcpress.com/Innovative-

Built-Heritage-Models-Editedcontributions-to-the-International/BalenVandesande/p/book/9781138498617.

[15] HeritageCARE, Survey of construction systems, type of damages and deterioration processes within the SUDOE territory, nt; HeritageCARE (Report of the Project Activity 1.1), 2017. http://heritagecare.eu/. 